\title{
Anne-Isabelle BouTON-TOUBOULIC (dir.), Magna voce. Effets et pouvoirs de la voix dans la philosophie et la littérature antiques
}

Paris, Classiques Garnier, 2021 (Kaïnon - Anthropologie de la pensée ancienne, 19), ISBN : 978-2-406-10660-9

Frédérique Woerther

\section{(2) OpenEdition}

Journals

Édition électronique

URL : https://journals.openedition.org/philosant/4178

DOI : $10.4000 /$ philosant.4178

ISSN : 2648-2789

Éditeur

Éditions Vrin

\section{Édition imprimée}

Date de publication : 15 décembre 2021

Pagination : 301-303

ISBN : 978-2-7116-3036-3

ISSN : 1634-4561

Référence électronique

Frédérique Woerther, « Anne-Isabelle воuтon-touboulıc (dir.), Magna voce. Effets et pouvoirs de la voix dans la philosophie et la littérature antiques », Philosophie antique [En ligne], 21 | 2021, mis en ligne le 23 mars 2021, consulté le 07 décembre 2022. URL : http://journals.openedition.org/philosant/4178 ; DOI : https://doi.org/10.4000/philosant.4178

\section{(c)}

Creative Commons - Attribution - Pas d'Utilisation Commerciale - Pas de Modification 4.0 International - CC BY-NC-ND 4.0

https://creativecommons.org/licenses/by-nc-nd/4.0/ 
peut y avoir ni ordre ni fin déterminée dans la production si le producteur n'a pas la connaissance ; toutefois celui-ci n'est pas la nature.

On le voit, la Physique est clairement lue par Simplicius comme déployant une philosophie de la nature néoplatonicienne, dont le commentaire livre de nombreux éléments essentiels; mais comme le rappelle l'auteur, il reste « qu'il ne faut pas réduire le commentaire à un simple corps de doctrine philosophique (...), le contenu philosophique ne peut être dissocié des dimensions exégétique, pédagogique et proprement spirituelle, qui constituent l'âme même du commentaire » (p. 18).

Philippe Soulier

Université de Nantes

Magna voce. Effets et pouvoirs de la voix dans la philosophie et la littérature antiques, sous la direction d'Anne-Isabelle Bouton-Touboulic, Paris, Classiques Garnier, 2021 (Kaïnon - Anthropologie de la pensée ancienne, 19), 473 p., ISBN : 978-2-406-10660-9.

En partie issu d'un colloque sur les «Effets de voix : la voix et les voix dans la pensée et la littérature romaines » qui s'est tenu en novembre 2014 à Lille, ce livre rassemble dix-huit contributions, classées selon quatre grandes catégories : I. Philosophie, II. Rhétorique et médecine, III. Acoustique, théâtre et littérature, IV. Linguistique, anthropologie, religion. Il comprend également une bibliographie (p. 385-423), un index des noms (p. 425-429), un index des passages cités (p. 431-457), un index des notions (p. 459-463) ainsi que le résumé de chaque essai (p. 465-470).

Un rapide survol de la table des matières et la lecture des premières lignes de cet ouvrage pourraient susciter de prime abord deux problèmes : $1^{\circ}$ nulle part l'éditrice ne définit strictement ce qu'elle entend par la « voix » qu'elle met au centre de son livre et qu'elle a choisie pour lui donner son titre, Magna Voce; $2^{\circ}$ les contributions qui y sont rassemblées obéissent somme toute à un classement très académique, qui ne semble pas remettre en question le cloisonnement artificiel entre les disciplines qui a si longtemps caractérisé le monde académique.

Mais en réalité, il n'en est rien.

Tout d'abord, Anne-Isabelle Bouton-Touboulic revendique l'idée qu'il s'agit ici d'appréhender « la voix comme une phénomène total, qui se diffracte en de nombreux domaines (musical, poétique, rhétorique, linguistique, philosophique, médical et religieux...) » (p. 9). C'est précisément ce dont il faut se réjouir, car c'est ce qui fait toute la force, toute la richesse, et tout l'intérêt de cet ouvrage. Les essais qui le composent parviennent en effet à créer de nouveaux réseaux de sens, à rassembler des approches particulières, à comparer des perspectives et des méthodes diverses. En invitant chaque contributeur à interpréter et définir le problème de la « voix » en fonction des impératifs propres à son domaine de spécialité, Anne-Isabelle Bouton-Touboulic a rendu possible le surgissement de questions et de méthodes empruntées à l'anthropologie, à l'histoire des religions, à la philosophie, à la linguistique, à l'analyse littéraire ou théâtrale - chacune de ces approches n'en excluant jamais une autre.

Ensuite, cette profusion de thématiques, de problématiques, de discussions qui naissent autour de l'idée de la « voix », ne débouche pas sur l'organisation artificielle de contributions hétérogènes, qui seraient paresseusement rangées selon les champs disciplinaires dont elles relèvent. Bien au contraire : derrière un classement thématique 
apparemment rigide (philosophie ; rhétorique et médecine ; acoustique, théâtre et littérature ; linguistique, anthropologie, religion) se dessine en réalité un savant, un subtil, un harmonieux ordonnancement, où courent de façon souterraine des thèmes très riches, qui seront définis et développés plus formellement ici, repris ailleurs avec de légères modulations.

Ainsi, l'approche linguistique, qui s'efforce de mettre au jour les distinctions sémantiques entre uox et les termes voisins (en latin), ou entre $\varphi \omega v \eta ́$ et les termes voisins (en grec) est convoquée avec plus ou moins de souplesse et de liberté selon les contributeurs et les perspectives qu' ils adoptent (et selon les auteurs anciens ou les corpus envisagés). Il faut à cet égard citer l'étude tout-à-fait passionnante de Frédérique Biville sur « Le bestiaire des voix humaines », emblématique de ce beau volume. Elle examine la question de la diversité des voix ; celle des frontières entre ars et natura (qu'on retrouvera par exemple dans le domaine de la rhétorique, avec la contribution de Charles Guérin), mais aussi la question des frontières entre humanité et animalité (soulevée également dans l'étude liminaire de Michel Crubellier consacrée à « Aristote. La voix humaine, l'écoute et l'entendement ») ; elle étudie également la valeur qu'il convient d'attribuer à la notion d'« imitation » (des animaux par les humains, des humains par les animaux), laquelle conduit à une problématique non seulement médicale (physiognomonique) déployée dans l'article de Vivien Longhi sur « Les pouvoirs de la voix dans la médecine grecque »- mais aussi littéraire et rhétorique de la «métaphore ».

Autre chapitre emblématique de cet enchevêtrement savant de thèmes qui parvient à tisser un réseau solide, serré, et harmonieux autour de la notion de voix : la contribution d'Anne-Isabelle Bouton-Touboulic sur Sénèque («Effets et méfaits de la voix chez Sénèque »). L'autrice reprend, dans la perspective sénéquienne, des questionnements de type philosophique sur la définition et la production de la voix - déployés dans le détail dans la contribution de Michel Crubellier dédiée à Aristote, comme on l'a dit, dans la contribution de Giulia Scalas consacrée à « La phonation et ses causes chez Épicure », ainsi que dans l'article de Thomas Bénatouil, qui est parvenu avec une clarté admirable et exemplaire à expliquer « L'analyse stoïcienne de la voix chez Diogène le Babylonien ». Le chapitre d'Anne-Isabelle Bouton-Touboulic établit également des liens étroits avec la thématique de la norme ( « Mauvais et bons usages de la voix », p. 95 sq.), qui sera reprise avec profondeur dans la belle étude de Charles Guérin, qui jette un regard si novateur sur le corpus longtemps négligé de Sénèque l'Ancien (« Les déclamateur latins et les variations de la norme vocale au ${ }^{\text {er }}$ siècle ap. J.-C. »), lorsqu'il examine la voix à travers la notion de « norme », qui charrie des valeurs aussi bien techniques (rhétoriques), qu'anthropologiques, médicales et littéraires (fictionnelles). Enfin, l'étude d'AnneIsabelle Bouton-Touboulic fait encore écho à l'étude de Vivien Longhi, consacrée à la médecine, dans la mesure où la « voix dans la psychagogie » (p. 95), et notamment la voix du maître de philosophie, n'est pas sans partager des points communs avec la « voix thérapeutique du médecin » (p. 173-178).

Les rapports entre la voix et l'espace constituent également l'une des thématiques, riches, novatrices, et fertiles qui traversent plusieurs chapitres de ce livre. L'étude de Gabrièle Wersinger (« Voix médiatisée, voix 'privées' ? Pour une topographie acoustique connective chez les anciens Grecs ») soulève la question de savoir si, « dans une communauté des affections médiatisées et de leurs expressions codifiées », il y a encore de la place pour «ce qu'on appelle la 'voix privée', par exemple l'expression phonique ou tonale d'une douleur, d'une joie ou d'une émotion personnelles » (p. 182), en observant, en déplaçant cette question sur le terrain de la topographie, puisque cette dichotomie voix publique/voix privée prend la forme d'une opposition, ou d'une tension, 
entre « une acoustique du joug et du cercle centré » d'une part, et « une acoustique de l'interaction et de la connexion » d'autre part (p. 191). L'espace et la voix, et leurs rapports, sont aussi au centre de l'étude de Marie-Hélène Garelli («Voix, personnage et espace dramatique dans les comédies de Plaute »), qui démontre en effet que, chez Plaute, loin de permettre l'identification d'un personnage, la voix agit plutôt comme le signe dramaturgique efficace des scènes de rencontre et participe à la construction de l'espace scénique. Voix et espace sont enfin également convoqués, à la faveur d'une subtile transition puisque l'on se situe désormais dans les espaces inaccessibles où domine le « numineux », dans l'article de Sébastien Barbara «Vox sine auctore. Vox et uoces du bois et d'ailleurs ».

La thématique de la pathologie constitue aussi l'un des fils rouges de Magna Voce, autour duquel se meuvent les chapitres de Charles Guérin (avec la notion de norme vocale, qui implique l'observation de ce qui n'est pas dans la norme), mais aussi l'étude, aussi exhaustive que limpide, de Florence Klein sur «'I $\chi \chi v o \varphi \omega v i ́ \alpha$. Les affect(at) ions vocales de Callimaque et quelques échos romains (Ovide, Perse) », où l'autrice examine l'hypothèse selon laquelle le bégaiement de Callimaque aurait été une façon de revendiquer le choix esthétique d'une poétique discontinue.

On pourrait encore citer la thématique de la technique, celle des rapports entre voix et musique...

Ces quelques exemples sont évidemment loin d'épuiser la richesse des parcours de lecture offerts dans ce volume. C'est qu'il importe de citer encore les articles de MariePierre Noël, sur « La voix de l'orateur et ses substituts dans les discours écrits chez Alcidamas, Isocrate et Aristote », qui propose une nouvelle analyse des rapports entre oral et écrit dans la rhétorique grecque des $\mathrm{IV}^{\mathrm{e}}$ - $\mathrm{V}^{\mathrm{e}}$ siècles avant J.-C.; de Sophie Conte, sur la notion à la fois politique et rhétorique de suanitas ( « La 'douceur' (suauitas) de la voix dans les Vacationes autumnales de Louis de Cressolles (1620) ») ; de Guy Lachenaud sur « La voix et les voix chez Plutarque. De la tribune au banquet »; d'Alain Deremetz sur «Les prestiges de la voix chez Apulée », où la voix de l'animal côtoie celle de l'homme, de l'orateur, et du philosophe; de Charles Guittard sur « Parole des dieux et dieux de la parole dans la religion romaine. Carmen et prophétie », qui, proposant une étude sur le carmen envisagé comme dialogue possible entre les hommes et les dieux, reçoit en retour un éclairage anthropologique dans la contribution de Sabine Crippa, qui examine les rôles des voix divines et humaines dans les contextes rituels des traditions polythéistes de l'Antiquité, et réfléchit notamment sur la notion d'« interférences vocales » («Interférences sonores entre les dieux et les hommes »). Enfin, François CassingenaTrévedy suggère, dans un dernier chapitre, de lire les Confessions d'Augustin comme une histoire de la voix («Les Confessions d'Augustin : une histoire sainte de la voix. De la voix du rhéteur à la voix de l'enfant »).

Il est impossible de rendre compte ici de toute la richesse, de toute la profusion, de toute l'intelligence de ce volume : son caractère hétéroclite et bigarré recèle en réalité une abondance réfléchie et harmonieuse. En invitant chaque lecteur à reconstruire son propre cheminement à travers ces dix-huit études, Magna Voce parvient à recréer réellement le monde des voix antiques. 